\title{
ARTICLE
}

Translational Therapeutics

\section{The tyrosine-kinase inhibitor sunitinib targets vascular endothelial (VE)-cadherin: a marker of response to antitumoural treatment in metastatic renal cell carcinoma}

\author{
Helena Polena ${ }^{1}$, Julie Creuzet ${ }^{1}$, Maeva Dufies ${ }^{2}$, Adama Sidibé $^{1}$, Abir Khalil-Mgharbel ${ }^{1}$, Aude Salomon ${ }^{1}$, Alban Deroux ${ }^{3}$, \\ Jean-Louis Quesada ${ }^{4}$, Caroline Roelants ${ }^{1}$, Odile Filhol ${ }^{1}$, Claude Cochet ${ }^{1}$, Ellen Blanc ${ }^{5}$, Céline Ferlay-Segura ${ }^{5}$, Delphine Borchiellini ${ }^{6}$, \\ Jean-Marc Ferrero ${ }^{6}$, Bernard Escudier ${ }^{7}$, Sylvie Négrier ${ }^{5}$, Gilles Pages ${ }^{8}$ and Isabelle Vilgrain ${ }^{1}$
}

BACKGROUND: Vascular endothelial (VE)-cadherin is an endothelial cell-specific protein responsible for endothelium integrity. Its adhesive properties are regulated by post-translational processing, such as tyrosine phosphorylation at site $Y^{685}$ in its cytoplasmic domain, and cleavage of its extracellular domain (sVE). In hormone-refractory metastatic breast cancer, we recently demonstrated that sVE levels correlate to poor survival. In the present study, we determine whether kidney cancer therapies had an effect on VEcadherin structural modifications and their clinical interest to monitor patient outcome.

METHODS: The effects of kidney cancer biotherapies were tested on an endothelial monolayer model mimicking the endothelium lining blood vessels and on a homotypic and heterotypic 3D cell model mimicking tumour growth. sVE was quantified by ELISA in renal cell carcinoma patients initiating sunitinib (48 patients) or bevacizumab (83 patients) in the first-line metastatic setting (SUVEGIL and TORAVA trials).

RESULTS: Human VE-cadherin is a direct target for sunitinib which inhibits its VEGF-induced phosphorylation and cleavage on endothelial monolayer and endothelial cell migration in the 3D model. The tumour cell environment modulates VE-cadherin functions through MMPs and VEGF. We demonstrate the presence of soluble VE-cadherin in the sera of mRCC patients $(n=131)$ which level at baseline, is higher than in a healthy donor group $(n=96)$. Analysis of sVE level after 4 weeks of treatment showed that a decrease in SVE level discriminates the responders vs. non-responders to sunitinib, but not bevacizumab.

CONCLUSIONS: These data highlight the interest for the sVE bioassay in future follow-up of cancer patients treated with targeted therapies such as tyrosine-kinase inhibitors.

British Journal of Cancer (2018) 118:1179-1188; https://doi.org/10.1038/s41416-018-0054-5

\section{INTRODUCTION}

Renal cell carcinoma (RCC) represents the 12th most frequent type of cancer and the $2 \%$ of all adult malignancies. ${ }^{1}$ Between 25 and $30 \%$ of the patients will develop metastatic renal cell carcinoma (mRCC) by the time they are diagnosed. Over the last 10 years three classes of targeted therapies have been developed, to treat mRCC. The first is multi-targeted tyrosine-kinase inhibitors (TKI) that include sorafenib, axitinib, pazopanib, and sunitinib. The second class is the mammalian target of rapamycin (mTOR) complex- 1 kinase inhibitors represented by temsirolimus (TEM), everolimus. The third class is the humanised anti-vascular endothelial growth factor (VEGF) monoclonal antibody, bevacizumab (BEV), and interferon $2 a$ (IFN). ${ }^{2,3}$

Since its approval in 2006 sunitinib (SUT) is a standard of care in the first line treatment of mRCC that has allowed an improved outcome. ${ }^{4-7}$ SUT is a multi-target TKI which belongs to the class of anti-angiogenic therapies that target the VEGF-signalling pathway. ${ }^{8}$ Despite significant improvements in survival, several reports have described acquired resistance to anti-angiogenic TKI after 6 months to 3 years of disease control. ${ }^{9-12}$ Thus, there is an urgent need for potential predictive biomarkers that could stratify patients who would benefit from treatment with SUT.

RCC is a highly angiogenic cancer. Angiogenesis involves the remodeling of endothelial cells (ECs), which acquire cell-cell contacts by homophilic interaction of vascular endothelial (VE)cadherin molecules expressed by neighboring cells. ${ }^{13}$ In normal healthy vessels ECs are tightly bound to each other, while in angiogenic vessels in tumours, ECs are stimulated by cytokines and growth factors in a process that leads to cell-cell destabilisation. ${ }^{14}$ Several cellular pathways are involved in these processes

\footnotetext{
${ }^{1}$ Univ-Grenoble Alpes, INSERM, CNRS, BIG-BCI Biology of Cancer and Infection, Grenoble F- 38054, France; ${ }^{2}$ Biomedical Department, Centre Scientifique de Monaco, Monaco, Monaco; ${ }^{3}$ Grenoble University Hospital, Division of Internal Medicine, Grenoble F-38043, France; ${ }^{4}$ INSERM, Unité 003, Clinical Investigation Center, Grenoble University Hospital, Grenoble F-38043, France; ${ }^{5}$ Unicancer, Centre de Lutte contre Le Cancer Léon Bérard, Lyon F-69008, France; ${ }^{6}$ Department of Clinical Research, Innovation and Statistics, Centre Antoine Lacassagne, Nice F-06000, France; ${ }^{7}$ Gustave Roussy Cancer Campus, Grand Paris, Villejuif F-94800, France and ${ }^{8}$ University of Nice Sophia Antipolis, Institute for Research on Cancer and Aging of Nice, CNRS UMR 7284, INSERM U1081, Centre Antoine Lacassagne, Nice F-06107, France

Correspondence: Isabelle Vilgrain (ivilgrain@cea.fr)

These authors contributed equally: Helena Polena, Julie Creuzet, and Maeva Dufies.
}

Received: 4 August 2017 Revised: 2 February 2018 Accepted: 20 February 2018

Published online: 22 March 2018 
through VE-cadherin targeting. Indeed, we have previously shown that the VEGF-induced-VE-cadherin phosphorylation contributes to the inhibition of the adhesive properties of ECs. ${ }^{15}$ This event occurs in vivo in mice in highly vascularised organs such as ovaries and uterus, during the angiogenic switch induced by hormones. ${ }^{16-18}$ Another mechanism involved in ECs destabilisation is the cleavage of the extracellular domain of VE-cadherin upon cytokines challenge such as TNFa ${ }^{19}$ and VEGF. ${ }^{20}$ Of major importance, a soluble form of VE-cadherin (SVE) has been detected in the bloodstream of patients with several diseases ${ }^{17,18}$ including breast cancer. Indeed, in metastatic breast cancer, we recently demonstrated that sVE was an independent prognostic factor for both progression-free survival and overall survival. ${ }^{21}$

The aim of the present study was to determine whether antiangiogenic treatment commonly used for the clinical care of mRCC had an effect on VE-cadherin post-translational modifications (i.e., phosphorylation and cleavage) and if so, whether such modifications were helpful to monitor patient outcome.

\section{MATERIALS AND METHODS}

\section{Reagents}

Recombinant human VEGF 165 was obtained from PromCell (Rocky Hill, NJ, USA). Sunitinib, temsirolimus, interferon 2aA, irinotecan, SN38 (the active metabolite of irinotecan), poly-2hydroxyethyl methacrylate (polyHEMA), sodium orthovanadate, and gelatin were purchased from Sigma-Aldrich (St Quentin Fallavier, France). CellTracker Green, collagen, Enhanced chemiluminescence detection reagents, the micro-bicinchoninic acid (micro-BCA) protein assay reagent kit were purchased from ThermoFisher Scientific (Courtaboeuf, France). Nitrocellulose was obtained from Schleicher \& Schuell BioScience (Perkin-Elmer Life Science, FRANCE).

\section{Antibodies}

We used the following commercially available antibodies including the mouse monoclonal antibody to human VE-cadherin (clone BV9) (Santa Cruz Biotechnology, SantaCruz, CA, USA), the monoclonal anti-phosphotyrosine antibody (clone 4G10) (Millipore Paris, France), the rabbit anti phosphoY ${ }^{685}$-VE-cadherin antibody (produced in the laboratory) and characterised in refs. ${ }^{17,18}$ and the mouse polyclonal anti- $\beta$ actin (clone AC-15) (Sigma-Aldrich, St Quentin-Fallavier, France). As secondary antibodies, we used the horseradish peroxidase-conjugated goat antimouse and anti-goat immonoglobulin G antibodies (Bio-Rad Laboratories, Marnes la Coquette, France).

Cell culture

Endothelial cells. Human Umbilical Vein Endothelial Cells (HUVECs) were grown and analysed as described by Vilgrain et al. $^{20}$

Renal cells. The renal carcinoma-derived cell line 786-O from American Type Culture Collection (ATCC, Manassas, USA) was grown in RPMI medium supplemented with $10 \% \mathrm{FBS}, 100 \mathrm{U} / \mathrm{mL}$ penicillin, $100 \mu \mathrm{g} / \mathrm{mL}$ streptomycin and $0.25 \mu \mathrm{g} / \mathrm{mL}$ amphotericin. Conditioned media from $10 \mathrm{~cm}$ dishes $\left(8 \times 10^{6}\right.$ cells) were collected after serum starvation, centrifuged at 14,000 r.p.m. to discard flotting cells, and concentrated on Centriprep Centrifugal Filter Units with an Ultracel YM-30 membrane (SODIPRO, Echirolles, France) and kept at $-80^{\circ} \mathrm{C}$ before use.

Zymography. Zymograms were performed as described in Lê et al. ${ }^{22}$ using bovine gelatin (final concentration $1 \mathrm{mg} / \mathrm{mL}$ ). Conditioned media from 786-O RCC cells were loaded onto a $12 \%$ acrylamide SDS-PAGE. After electrophoresis at $0.02 \mathrm{~A}$ for $2 \mathrm{~h}$ $30 \mathrm{~min}$ in Tris/ $\mathrm{HCl} 25 \mathrm{mM}$ glycine $192 \mathrm{mM}$ buffer, the gelatin gels were then successively washed at room temperature (RT) under agitation twice in $100 \mathrm{~mL} 2.5 \%$ Triton $\mathrm{X}-100$ to remove SDS for 30 min, then in $50 \mathrm{mM}$ Tris/HCL, pH 8.5 containing $200 \mathrm{mM} \mathrm{NaCl}, 10$ $\mathrm{mM} \mathrm{CaCl}_{2}$ for $15 \mathrm{~min}$ and overnight (ON) in the same buffer at $37^{\circ}$ C. After three washing steps of $10 \mathrm{~min}$ in water, gels were successively stained with Coomassie blue for $1 \mathrm{~h}$ at RT before being destained in $100 \mathrm{~mL} 20 \%$ methanol $10 \%$ acetic acid for 30 min and then in water.

Preparation of cell extracts, electrophoresis, and immunoblotting. Cell lysates, and immunoblots were prepared and analysed as described previously by Vilgrain et al. ${ }^{20}$

Generation of spheroids. Confluent monolayers ECs were incubated for $30 \mathrm{~min}$ with $10 \mu \mathrm{M}$ of the CellTracker Reagent. After $4 \mathrm{~h}$ of incubation, ECs were trypsinised and suspended either alone or with 786-O RCC cells (1:1) onto poly-HEMA-coated plates ( $20 \mathrm{mg}$ / $\mathrm{mL}$ ). Under these conditions all suspended cells contributed to the formation of a single spheroid. After $72 \mathrm{~h}$ of culture, the spheroids were collected, transferred onto a collagen gel $(4 \mathrm{mg} / \mathrm{mL})$, and stimulated with either VEGF alone $(50 \mathrm{ng} / \mathrm{mL})$, or in combination with mRCC therapies. RCC and ECs migration from the spheroid was imaged with a Zeiss AxioObserver Z1. After $24 \mathrm{~h}$ of culture, the position of each EC $(n=500)$ was manually recorded using the ImageJ Cell-Counter plugin (http://rsbweb.nih.gov/ij/plugins/cellcounter.html) (http://imagej.nih.gov/ij/). $X$ and $Y$-values were then imported in a customised Python (https://www.python.org) script, which we created to calculate the Euclidean distance of each cell to the centre of the spheroid and the mean distance of the cells to the centre (Supplementary Figure S1).

Enzyme linked immunosorbent assay (ELISA). ELISA for soluble VEcadherin was performed as described in Vilgrain et al. ${ }^{20}$

\section{Patients' blood samples}

Healthy donors $(H D)$. Sera from healthy donors eligible to give their blood were collected at "Etablissement Français du Sang" (EFS, Grenoble, France) and kept frozen at $-80^{\circ} \mathrm{C}$ until use. The study protocol was approved by the EFS and written informed consent was obtained from each participant.

Patients with metastatic renal cell cancer. Sera from patients with metastatic renal cell cancer receiving SUT or TEM + BEV or BEV + IFN were obtained from 2 clinical trials (TORAVA and SUVEGIL) approved by local ethics committee. Written informed consent was obtained from each patient before enrollment. Patients' characteristics are described in Supplementary Table 1.

TORAVA. (clinicaltrials.gov NCT00619268-sponsor: Centre Léon Bérard, Lyon, France). Patients'recruitment and data-collection methods of the TORAVA trial were described in (Negrier et al. ${ }^{44}$ ). Blood samples were collected at three different time points: at baseline prior to treatment (Day 0), 2 weeks (Day 15), and 5-6 weeks (Day 40) after the beginning of the treatment. Samples were stored at $-196^{\circ} \mathrm{C}$ in liquid nitrogen after collection in the Biological resource centre BB-0033-00050 of the Centre Léon Bérard, Lyon, France.

SUVEGIL. (clinicaltrials.gov NCT00943839- sponsor: Centre Antoine Lacassagne, Nice, France). Each cycle lasted 6 weeks in the absence of disease progression or unacceptable toxicity and was organised in the protocol of four consecutive weeks of oral administration of sunitinib malate $(50 \mathrm{mg} /$ day $)$ followed by 2 weeks of rest. Blood samples were collected before the treatment and at the end of the fourth week of SUT treatment. ${ }^{23}$

Statistical analysis

For cell analysis. All of the experiments were repeated at least three times. Western blot bands have been analysed to 
A

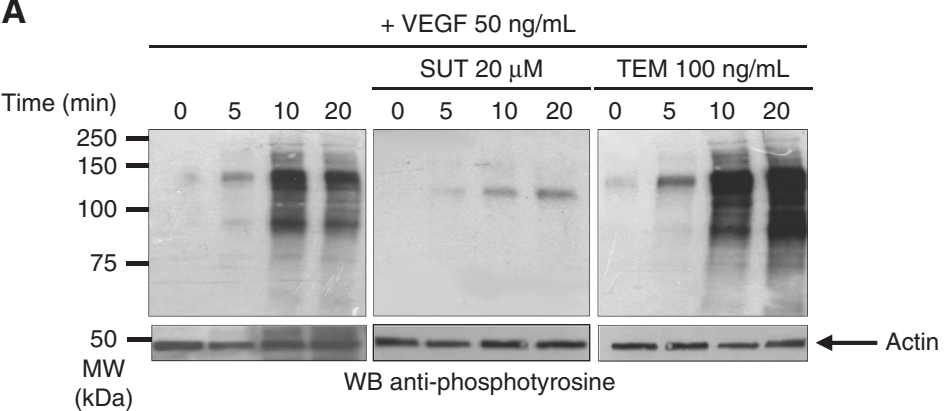

B

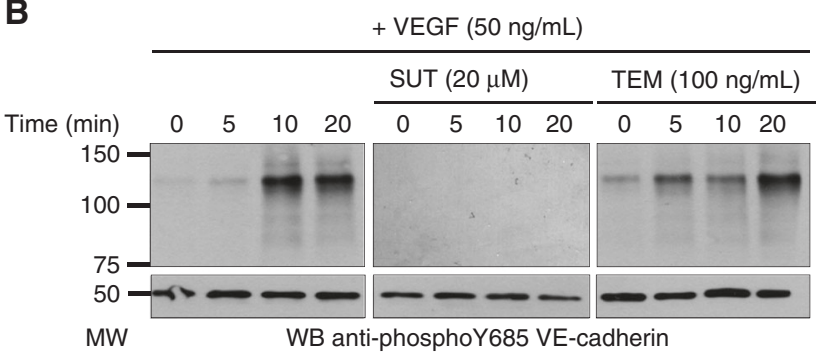
(kDa)

D

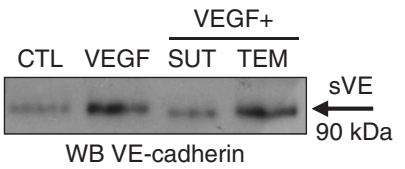

C

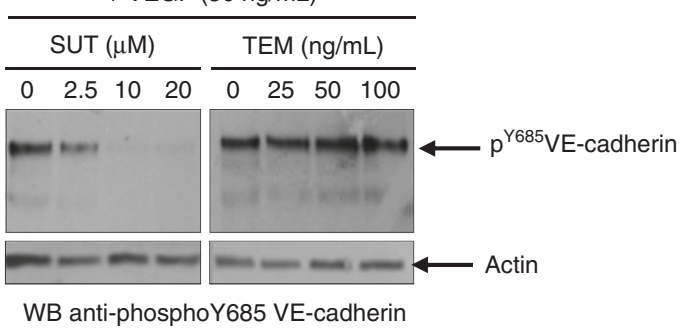

$\mathbf{E}$

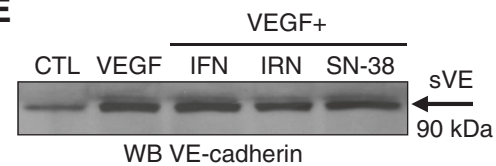

Fig. 1 Sunitinib impairs VEGF-induced VE-cadherin tyrosine phosphorylation and cleavage: primary ECs were grown 3-4 days to reach confluency $\left(3 \times 10^{6}\right.$ cells). After $3 \mathrm{~h}$ of serum starvation, ECs were stimulated by VEGF alone $(50 \mathrm{ng} / \mathrm{mL})$ or in the presence of SUT $(20 \mu \mathrm{M})$ or TEM $(50 \mathrm{ng} / \mathrm{mL})$ during indicated times. Lysates were analysed by SDS-PAGE on $10 \%$ polyacrylamide gels and western blotting $(5 \mu \mathrm{g}$ of total protein lysate/lane). a Analysis of phosphotyrosine-containing protein pattern using the anti-phosphotyrosine antibody $4 \mathrm{G} 10$. $\mathbf{b}$, c Analysis of phospho$Y^{685}$ VE-cadherin d. Conditioned media from untreated ECs (CTL), or VEGF-stimulated-ECs or VEGF-stimulated-ECs treated with SUT or TEM for 20 min (same amount of cells in each condition) were collected, centrifuged to discard floating cells, and concentrated on Centriprep Centrifugal Filter Units with an Ultracel YM-30 membrane. Volume of $5 \mu \mathrm{L}$ of concentrated medium of each condition was analysed by SDS-PAGE and western blotting using the monoclonal anti-VE-cadherin antibody (BV9 clone). e Same experiment as in d with VEGF-treated ECs in the presence of either INF $\alpha 2 \mathrm{~A}(7.5 \mathrm{ng} / \mathrm{mL})$, Irinotecan $(\mathrm{IRN}, 10 \mu \mathrm{M})$ and SN-38 $(10 \mu \mathrm{M})$. Results are representative of three independent experiments

densitometry using Image $J$ software and data are expressed in arbitrary units as the mean \pm SD of at least three identical experiments (same number of cells in each dishes). Student's $t$ test was used to compare the means of data from two experimental groups. One-way ANOVA was used when three or more experimental groups were compared, and analysis of significance was performed using Tukey's range test $\left({ }^{*} P<0.05\right.$; $\left.{ }^{* *} P<0.01 ; * * * P<0.001\right)$. For all tests, $P$-values $\leq 0.05$ were considered significant.

For sVE analysis in blood sample. The non-parametric Mann-Whitney U-test was used to compare sVE levels between mRCC patients and the HD group. Wilcoxon and Fisher Exact tests were used to evaluate the relationship between sVE levels and clinical response to treatment. Progression-free survival was defined as the time from inclusion to the date of first documentation of progression or the date of death or date of last follow-up. Survival data were assessed by the Kaplan-Meier method. Statistical analysis was performed using Stata release 11.0 (StataCorp, College Station, TX)—PC software. For all tests, $P$-values $<0.05$ were considered statistically significant.

\section{RESULTS}

VE-cadherin is a target for Sunitinib

A confluent ECs monolayer has been used to mimic the vascular endothelium lining all blood vessels. The effect of SUT and TEM on ECs was assessed by analysing tyrosine phosphorylated-proteins as a sensitive readout of the activation of the VEGF/VEGFR signalling pathway. As shown in Fig. 1a, the pattern of VEGFinduced time dependent-tyrosine phosphorylated protein (left panel) was reduced by SUT (middle panel) but not affected by TEM (right panel). The time-dependent-VE-cadherin $\mathrm{Y}^{685}$-phosphorylation induced by VEGF (Fig. 1b, left panel) was no longer detectable after SUT treatment but remained constant after TEM treatment (Fig. $1 \mathrm{~b}$, middle and right panels, respectively). Downregulation of VEGF-induced-VE-cadherin $Y^{685}$ phosphorylation was readily detectable at a SUT concentration as low as $2.5 \mu \mathrm{M}$ (Fig. 1c, left panel); whereas, the increasing concentrations of TEM (from 0 to $100 \mathrm{ng} / \mathrm{mL}$ ) had no effect (Fig. 2c, right panel). Because SUT is a $\mathrm{TKI}$, and TEM is a serine-threonine kinase inhibitor, these results are in agreement with the specific effects of these molecules. The destabilisation of ECs after drug treatment was analysed by the release of SVE in the ECs conditioned media. As shown in Fig. 1d, VEGF-induced SVE release was no longer detected with SUT treatment, while it was still detectable with TEM treatment. Other molecules commonly used in cancer treatment, such as interferon a2A (IFN), irinotecan (IRN), and its active metabolite SN-38, did not affect VEGF-induced sVE release (Fig. 1e). Taken together, these results suggest that the release of SVE from ECs might represent a specific readout of the effects of TKI on ECs, independently of their effects on cancer cells.

Effect of kidney cancer biotherapies on three dimensional (3D) spheroids of tumour cells and ECs

Because in vitro $3 D$ cell cultures reflect the complex in vivo microenvironment, we next aimed at studying the effects of kidney cancer biotherapies on the sprouting of either one-cell 

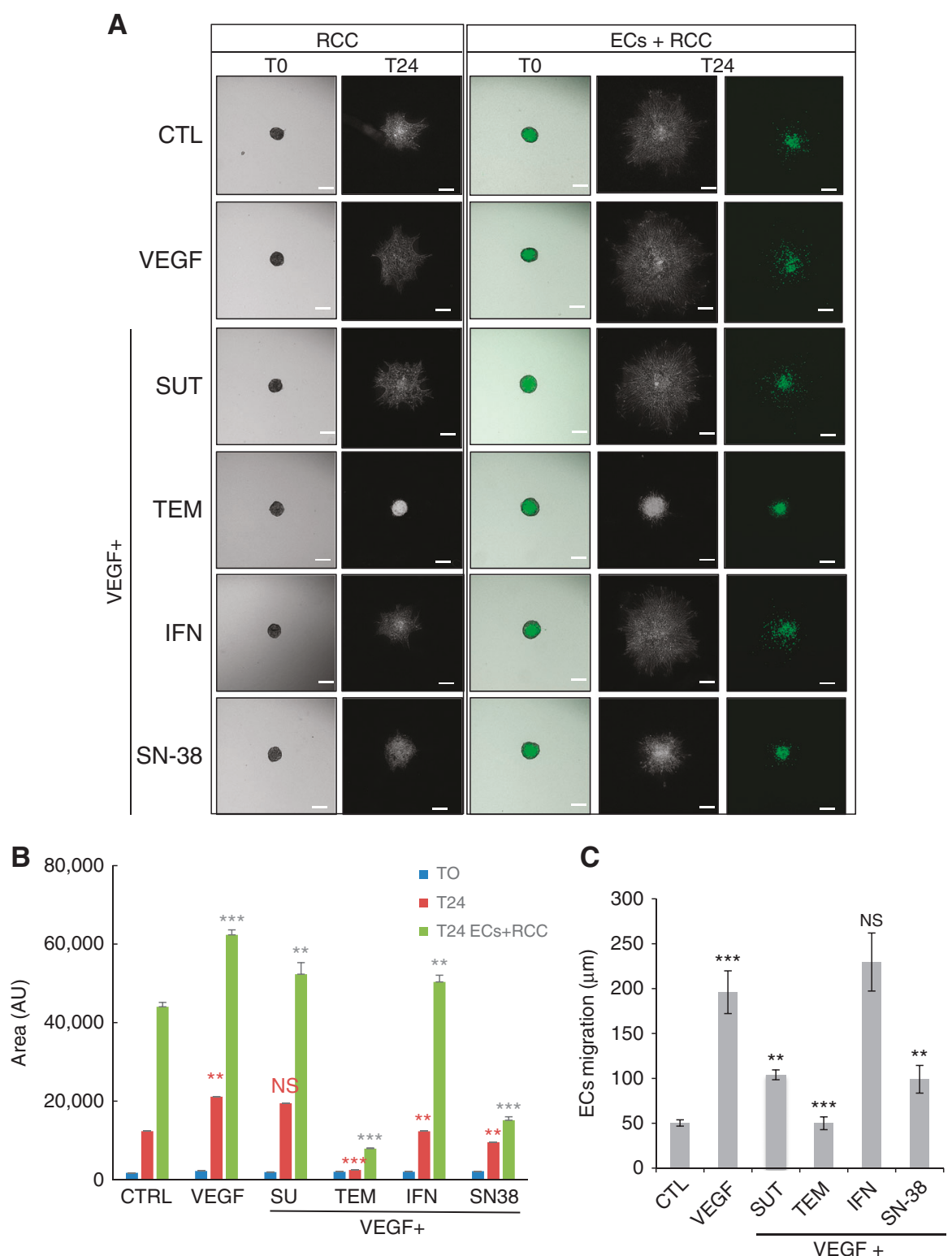

Fig. 2 SUT impairs cell invasion and migration in heterotypic spheroids. a Representative images of spheroids formed from 786-O (RCC) cells alone at the time of their incorporation into collagen gel (T0) and $24 \mathrm{~h}$ later (T24), or RCC + ECs at time 0 and $24 \mathrm{~h}$ later. b Area of the spheroids was quantified using Image J software (National Institutes of Health). c Quantification of ECs migration from spheroids co-cultured with RCC using the measurement of the Euclidean distance of each EC $(n=500)$ from the centre of the spheroid after $24 \mathrm{~h}$ of culture. Results are representative of three independent experiments. Values are the mean \pm SD of the migration distance in $\mu \mathrm{m}$. Statistical significance is indicated by the number of asterisks $\left(^{*}\right)$

type spheroid containing tumour RCC or a two-cell type co-culture spheroids containing tumour RCC and ECs (labeled with CellTracker). Figure $2 a$ shows representative images of spheroids at the time of their incorporation into collagen gel (T0) and $24 \mathrm{~h}$ later (T24). The spheroid area was then quantified at both time of culture upon basal conditions (CTL) or VEGF challenge either alone or in combination with the other indicated drugs. Figure $2 \mathrm{~b}$ shows that within $24 \mathrm{~h}$ the size of the RCC-spheroids expanded sevenfold in CTL conditions $(P<0.001)$ and 12 -fold upon VEGF challenge $(P$ $<0.001$ ), which is consistent with the presence of VEGF receptors on tumour cells. ${ }^{24}$ TEM, IFN, and SN-38 significantly decreased tumour spheroid expansion, which is in agreement with the antitumour effect of these molecules. ${ }^{25-27}$ In contrast, no significant effect of SUT on RCC spheroids was observed, which is in agreement with previous data showing that SUT did not affect RCC proliferation at concentrations that inhibit RTK signalling. ${ }^{8}$
In RCC-ECs 3D structures, spheroids expansion was higher than RCC spheroids in control conditions (areas: 44,038.33 \pm 1108.01 vs. $21,156 \pm 1106$, respectively, $P<0.001$ ) suggesting a cooperative effect of both cell types to the spheroid expansion. The expansion of the spheroid was stimulated by VEGF but weakly affected by SU and INF (-20\%) and strongly impaired by TEM and SN-38 (-80\%). The migration of ECs from the centre of the co-culture spheroids was then determined (Fig. 2c). After $24 \mathrm{~h}$ of culture at baseline, the migration of ECs was $50.33 \pm 3.51 \mu \mathrm{m}$. VEGF alone stimulated the ECs migration by four times $(P<0.001)$ with an average of migration of $196 \pm 23.8 \mu \mathrm{m}$, while this VEGF-induced ECs migration was partly blocked by SUT $(104 \pm 5.50 \mu \mathrm{m}$, or $53 \%$ of VEGF response), and SN-38 (99 $\pm 15.39 \mu \mathrm{m}$, or $50 \%$ of VEGF response), and was completely blocked by TEM $(50 \pm 7.09 \mu \mathrm{m}$ or control value), but unchanged by IFN $2 a(229.66 \pm 32.33 \mu \mathrm{m}$, or $117 \%$ of VEGF response). These results demonstrate that IFN did not impair 

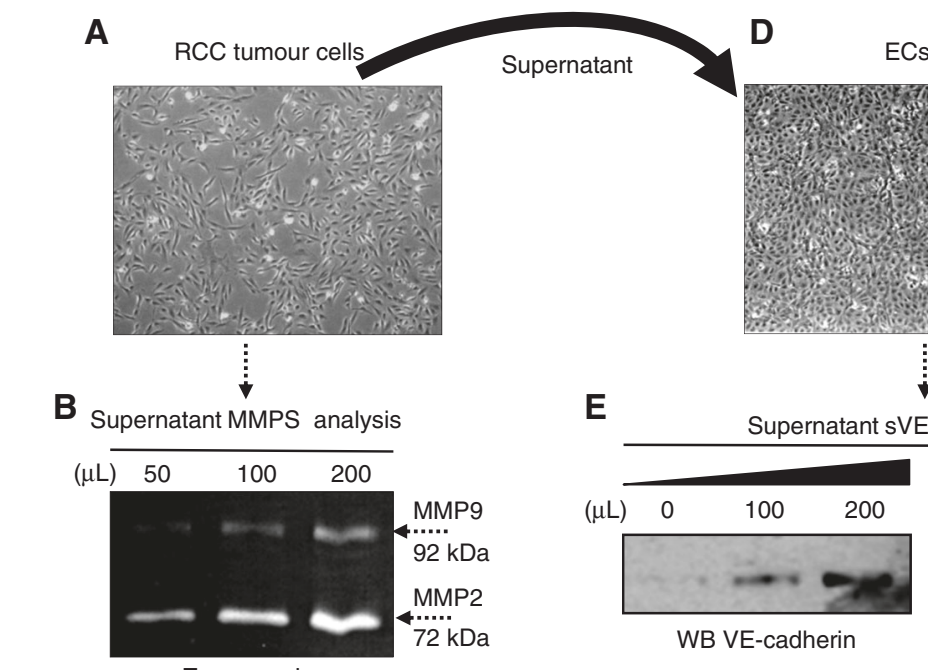

WB VE-cadherin

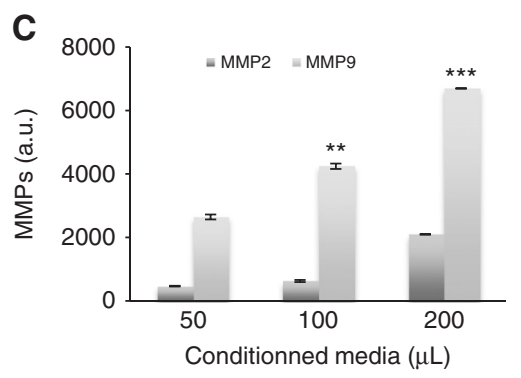

$\mathbf{F}$

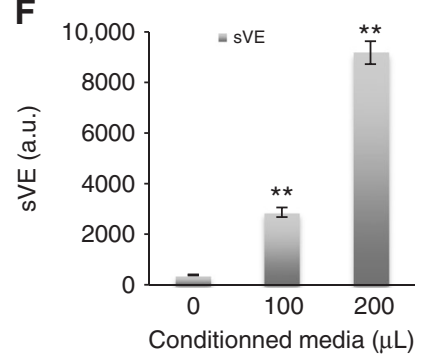

G

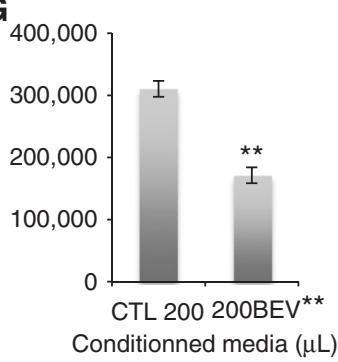

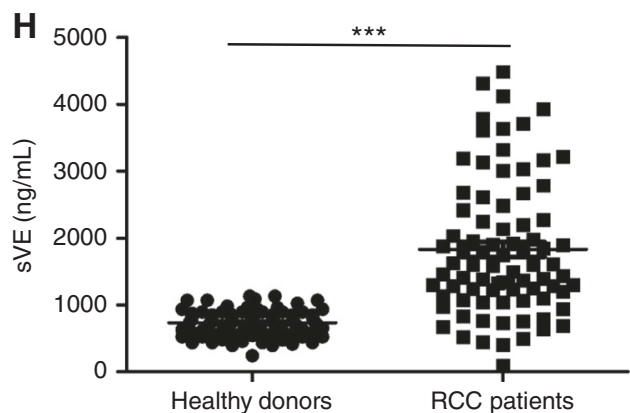

Fig. 3 Effect of RCC tumour microenvironment on VE-cadherin cleavage: elevated levels of sVE in mRCC patients: a Phase contrast microscopy of RCC cells in culture. b Analysis of RCC culture media by zymography showed the presence of MMP2 and MMP9 activities. c The relative amounts of MMPs were measured by densitometry of autoradiographs using ImageJ software (National Institutes of Health). $\mathbf{d}$ Phase contrast microscopy of ECs in culture. e Release of sVE by ECs upon RCC conditioned media treatment ( 20 min) (left panel) alone or RCC conditioned media pre-incubated or not with bevacizumab (BEV) $(5 \mu \mathrm{g} / \mathrm{mL})$ (right panel). $\mathbf{f}, \mathbf{g}$ The relative amounts of sVE were measured by densitometry of autoradiographs using ImageJ software (National Institutes of Health). Results are representative of three independent experiments. Values are the mean \pm SD. $\mathbf{h}$ sVE levels in healthy donors $(n=96)$ and mRCC patients from the clinical trial TORAVA $(n=115)$ at diagnosis $($ Day 0, D0) was performed by ELISA. Statistical significance is indicated by the number of asterisks $\left(^{*}\right)$

ECs migration in this 3D co-culture model, but SUT, SN-38 and TEM had a strong inhibitory effect. Taken together, these results indicate that the 3D model is a useful tool to detect the effect of anti-tumour drugs on several cell types including ECs.

RCC tumour microenvironment induces the release of SVE The differences in invasion process observed in RCC-ECs coculture spheroids vs. RCC spheroids, led us to determine whether RCC cells (Fig. 3a) could activate ECs and destabilise adherens junctions by affecting VE-cadherin function. We thus analysed potential RCC (Fig. 3a) secreted inducers of sVE release such as matrix metalloproteinases (MMPs), ${ }^{28}$ using the zymography technique (Fig. 3b). Two MMPs were identified based on their respective molecular weight corresponding to MMP9 (92 kDa) and
MMP2 (72 kDa) Analysis of the relative amount of enzyme in the tumour cell conditioned medium showed a dose-dependent expression of MMPs activities with a predominant activity for MMP2 (Fig. 3c). When applied to ECs (Fig. 3d), the tumour cell conditioned media-induced the release of SVE in a dosedependent manner within 20 min (Fig. 3e, f)). This result is consistent with our previous results showing that TNF-induced VEcadherin cleavage was mediated by several proteinases. ${ }^{19}$ Because RCC are known to secrete VEGF, ${ }^{29}$ we wondered whether VEGF was involved in this process. Thus the RCC conditioned medium was pre-treated or not with bevacizumab (BEV) for $30 \mathrm{~min}$ prior to its addition onto ECs. As shown in Fig. 3g, the release of sVE from ECs was significantly decreased when RCC conditioned medium that was pre-treated with BEV. Taken together, these results are 
A

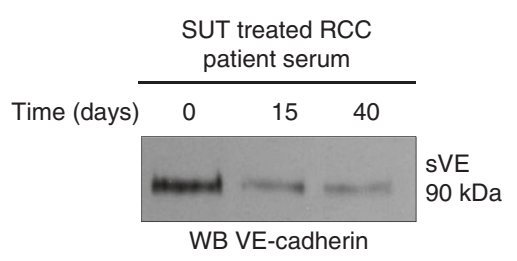

C

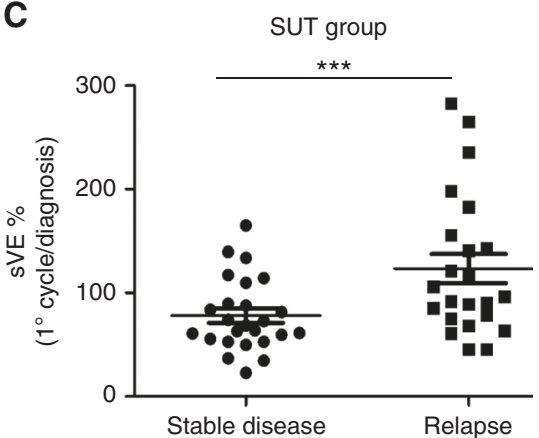

B

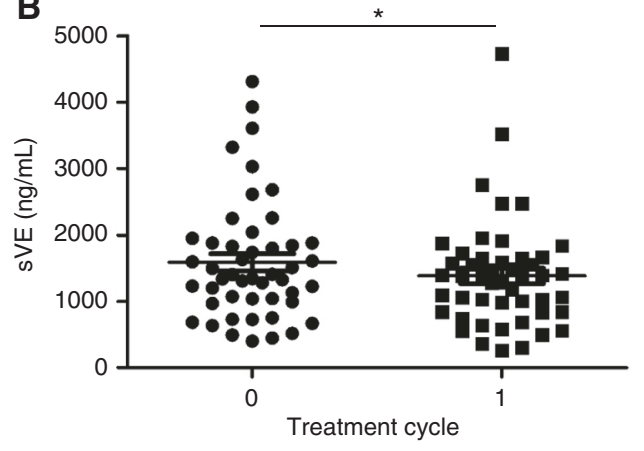

D

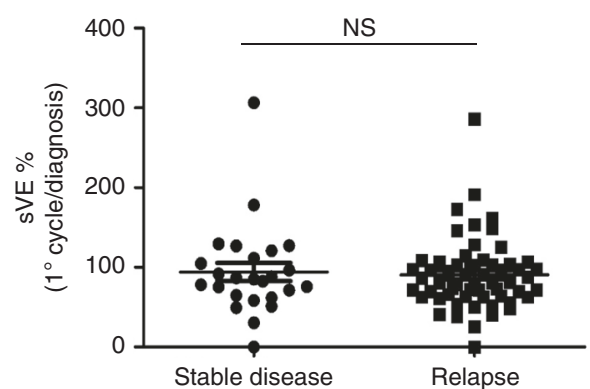

Fig. 4 Analysis of sVE- levels in mRCC patients after the first cycle of treatment. a Serum from mRCC patient treated with SUT was analysed for sVE-cadherin level using the monoclonal anti-VE-cadherin antibody. b sVE levels in mRCC patients after the first cycle of treatment. c Variations of sVE expressed as \% of the initial concentration at diagnosis (level of sVE after the first cycle/level of sVE at diagnosis $\times 100$ ) in SUT group with stable disease and relapse. $\mathbf{d}$ Variations of sVE expressed as $\%$ of the initial concentration at diagnosis (level of sVE after the first cycle/level of SVE at diagnosis $\times 100)$ in BEV group with stable disease and relapse. Statistical significance is indicated by the number of asterisks $(*)$

consistent with a role of the tumour microenvironment on the modulation of VE-cadherin functions and ECs behavior in the tumour.

sVE level is higher in MRCC patients at diagnosis $(n=131)$ than in healthy donors group $(n=96)$

Because the blood level of sVE has never been reported in $\mathrm{mRCC}$ patients, we analysed sVE level by ELISA in a cohort of mRCC patients from the TORAVA and SUVEGIL clinical trials $(n=131)$ that was compared to a group of healthy donors (HD; $n=96$ ). The description of mRCC patients is illustrated in Supplementary Table 1. The median level of sVE was significantly higher in $\mathrm{MRCC}$ patients (1831 ng/mL [min-max: 90-4484]) than in the HD group (731.0 ng/mL [min-max: 240-1133], $P<0.001$ ) (Fig. 3h). As mRCC is a highly vascularised cancer, we hypothesised that SVE might represent a potential new biomarker of the angiogenic processes involved in this type of cancer.

SUT treatment modulates sVE level in MRCC patients

We next determined the effect of SUT treatment on the blood level of sVE in mRCC patients. Figure $4 a$ shows a western blot analysis of serum samples from one patient before treatment (Day 0 ), after 2 weeks (D15) and after 6 weeks (D40) of SUT treatment. Interestingly, the $90 \mathrm{kDa}$ extracellular domain of VE-cadherin detected in the blood sample is decreased by the treatment for this patient. (Fig. 4a). To further confirm this observation, sVE ELISA was then performed for all mRCC patients from the two clinical trials (TORAVA and SUVEGIL, $n=48$ ) at diagnosis and after one cycle of treatment. As a result, we found that the sVE level was significantly decreased after the first cycle of treatment in patients with stable disease (Fig. 4b and Supplementary Figure S2 A) but not in patients who relapsed (Supplementary Fig S1 B). In the BEV group, sVE level after the first cycle was not predictive of the response to the treatment (Figure S2 C, D). We next calculated for each patient the variation of SVE after one cycle of SUT treatment as a $\%$ of the initial level at diagnosis (level of sVE after the first cycle/level of sVE at diagnosis $\times 100$ ). Patients with a stable disease had globally a decreased level of SVE after one cycle of SUT treatment (median ratio $78 \%$ corresponding to $22 \%$ of decrease) whereas SVE level continued to increase in patients who relapsed after treatment (median ratio $122 \%$ corresponding to $22 \%$ of increase) (Fig. $4 c, P=0.005$ ). sVE variation level was not significantly different in the two groups of patients who received BEV treatment (Fig. 4d) $P=0.7469$ ).

Analysis of sVE variations and clinical outcome

SVE variations and progression-free-survival were assessed by the Kaplan-Meier method, using a cutoff of $120 \%$ in mRCC patients treated with SUT alone, and in the BEV group. As illustrated in Fig. 5a, patients with an increased level of sVE after SUT treatment (sVE variation $>120 \%$ ) had a poorer outcome (median PFS 8.2 months) than patients with decreased sVE level (sVE variation $<120 \%$-median PFS: $=20.1$ months, $P=0.0097$ ). We next analysed SVE levels in patients from TORAVA clinical trial treated either with BEV plus IFN or BEV plus TEM. As a result, we found that the variation of sVE levels after the first cycle of treatment was not predictive of the response to treatment (Fig. 5b). The median PFS was 16.7 months and 10.9 months for patients treated with SUT or BEV, respectively (Supplementary Figure S3). Taken together, these results suggest that SVE is a biomarker of response to SUT therapy in $\mathrm{mRCC}$.

\section{DISCUSSION}

Several inhibitors of VEGF-signalling pathways are in clinical use for mRCC management. Because VEGF modulates VE-cadherin 
A

SUT group

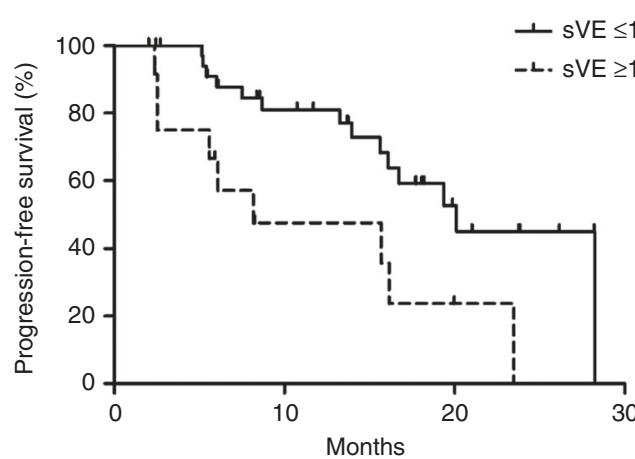

\begin{tabular}{|l|r|}
\hline Ratio sVE & $\begin{array}{r}\text { Median } \\
\text { (mo }\end{array}$ \\
\hline$<120 \%$ & \\
\hline$>120 \%$ & \\
& \\
& \\
Hazard ratio & 0,2452 \\
Ratio \\
$95 \% \mathrm{Cl}$ of ratio & 0.08452 to \\
& 0.7114 \\
\end{tabular}

B

BEV group

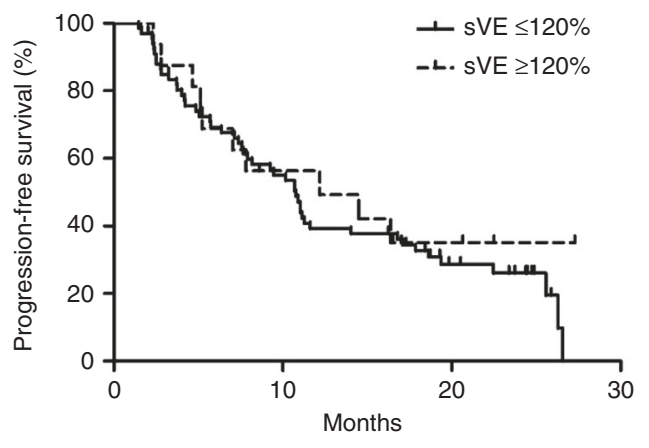

$P=0.4831$

\begin{tabular}{|c|c|}
\hline Ratio sVE & $\begin{array}{c}\text { Median survival } \\
\text { (months) }\end{array}$ \\
\hline$<120 \%$ & 10.8 \\
\hline$>120 \%$ & 12.2 \\
\hline zard ratio & 1,256 \\
\hline
\end{tabular}

$95 \% \mathrm{Cl}$ of ratio $\quad 0.6646$ to 2.372

Fig. 5 Relationship between sVE-cadherin levels and PFS of mRCC patients: a-c Kaplan-Meier analysis of PFS of patients with RCC. PFS was calculated from patient subgroups with variations of sVE expressed as $\%$ of the initial concentration at diagnosis (level of sVE after the first cycle/level of sVE at diagnosis $\times 100$ ) that were less or greater than a cutoff ratio at $120 \%$, for SUT group trial (a), (b) BEV group. Statistical significance $(P$-value) and the time of the median disease free are indicated

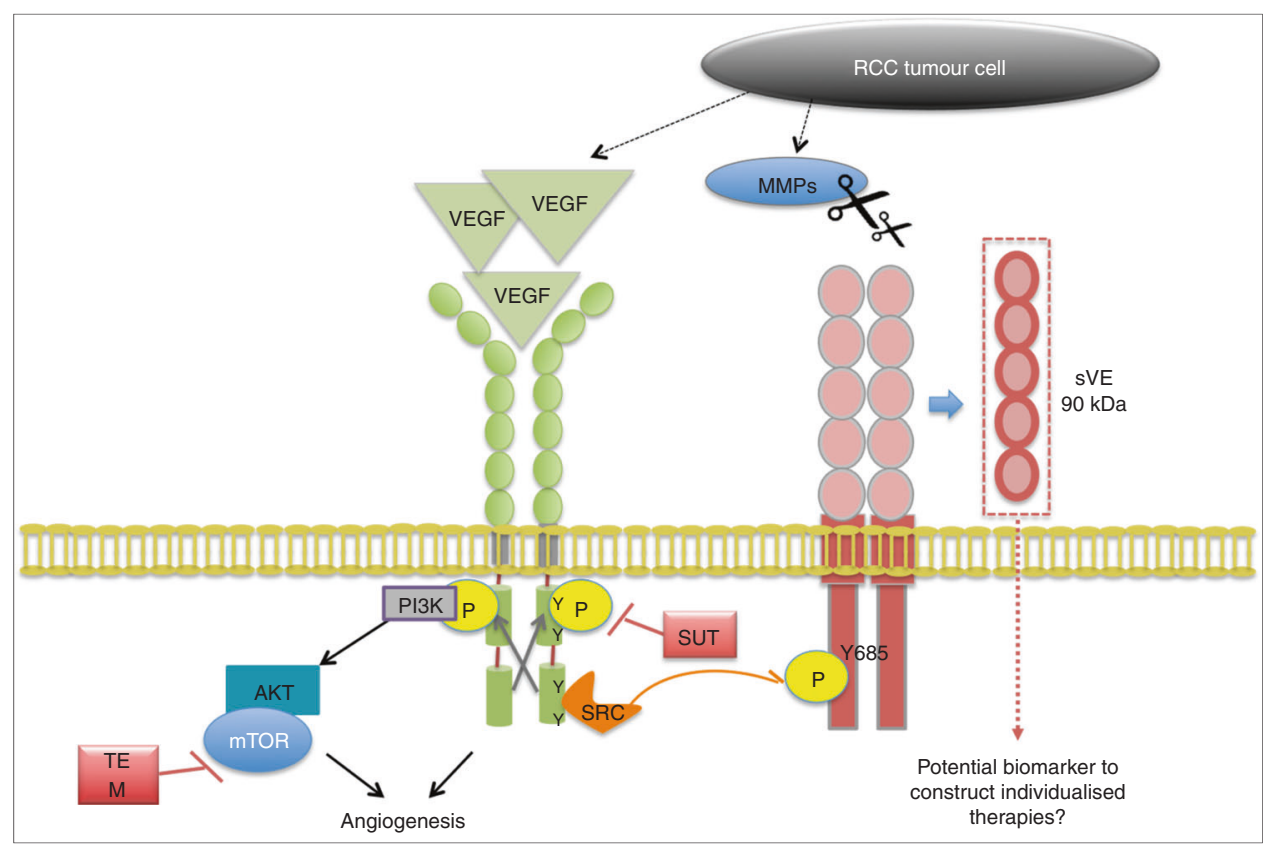

Fig. 6 Proposed mechanisms of microenvironment-induced VE-cadherin structural modifications in kidney cancer. This working hypothesis was mainly built from our results obtained using in vitro EC cultures and VEGF as the major actor in angiogenesis. ${ }^{15,20}$ The involvement of other cytokines in these processes cannot be excluded since we previously showed that TNF $\alpha$ induced sVE release ${ }^{19}$ 
structural modifications, in this study we determined the effect of kidney cancer therapies on VE-cadherin functions and its potential clinical interest.

Using a 2D ECs monolayer model to mimic the human endothelium, we studied the early effects of inhibitors of VEGF signalling on VE-cadherin. Among the compounds tested, we demonstrate for the first time that the tyrosine-kinase inhibitor SUT is the only molecule able to block VEGF-induced-sVE release. These observations are in agreement with our previous results obtained using PP2, a Src kinase inhibitor, or Genistein, a large spectrum TKI. ${ }^{19}$ These results are consistent with the specific effects of the inhibitors of VEGF signalling such as TEM, a serinethreonine kinase inhibitor, and IFN, IRN, and SN-38 which are not kinase inhibitors.

By using a 3D homotypic and heterotypic cell culture model to mimic tumour growth we showed that RCC spheroids growth was inhibited by TEM, IFN $2 a$, and SN-38, which is in agreement with their antitumour activity. Interestingly RCC and ECs co-culture spheroids showed an increased tumour cell invasion, especially upon VEGF stimulation. ECs migration were strongly impaired when VEGF-stimulated spheroids were incubated in the presence of SUT. This result is consistent with a previous study showing that SUT is active in vitro against activated ECs via downregulation of VEGF-Receptor $2 .^{30}$ Interestingly, we found here that the $2 \mathrm{D}$ and the $3 \mathrm{D}$ models did not provide the same information. Indeed, on the 2D endothelial monolayer, only SUT impaired SVE release which is a process related to ECs migration. In the 3D co-culture spheroids, both SUT, TEM, and SN-38 inhibited ECs migration. These results suggest that the heterotypic spheroid model is a useful model to detect the impact of new anticancer drugs on ECs and to anticipate the potential side effect of new molecules on the vascular system in addition to their anti-tumour activity. The results obtained with the EC monolayers, which reveals the early events involved in VEGF-signalling pathway $(20 \mathrm{~min})$, did not identify the potential side effects of these molecules. Nonetheless, it has been reported that after $24 \mathrm{~h}$ of treatment, TKIs can inhibit ECs growth, with $\mathrm{IC}_{50}$ around $2 \mu \mathrm{M}$. TEM also reduced ECs growth by $50 \%$, at concentrations as low as 0.05 or $0.5 \mathrm{nM}$ after 48 or $72 \mathrm{~h}$, respectively. ${ }^{31} \mathrm{SN}-38$ was reported to have anti-angiogenic properties at concentrations ranging from 0.01 to $1 \mu \mathrm{M}$ (here used at $10 \mu \mathrm{M}) .{ }^{32}$ We used in our study the same concentrations in $2 D$ and $3 D$ cultures (e.g., $50 \mathrm{ng} / \mathrm{mL}$ ), which are the average concentration of SUT found in blood patients after cycle 1 which is in a direct contact with the endothelium (data not shown). It can be suggested that the long term effect of these concentrations of TKI found in blood might have other side effects on endothelium from healthy vessels. Further studies will be required to determine whether SVE is a biomarker of vascular integrity after biotherapies treatment and possibly a biomarker of toxicities.

We have found that tumour cells could activate ECs through VEGF and MMPs secretion, which is in agreement with previous reports (Gialeli et al., 2010) and with our data showing that MMPs are involved in TNFa-induced VE-cadherin cleavage. ${ }^{19}$ It can be assumed that MMPs found in the tumour microenvironment could participate in ECs remodeling by inducing VE-cadherin cleavage, as it was already shown for the cleavage of several cell surface proteins. ${ }^{33}$ Our working hypothesis is illustrated in Fig. 6 . Tumour cells secrete cytokines such as VEGF and proteases like MMPs. Upon binding to its receptors, VEGF induces VE-cadherin tyrosine phosphorylation at site Y685 mediated by Src which is a covalent modification that changes VE-cadherin conformation. The protein is thus more sensitive to cleavage by proteases and the soluble form of VE-cadherin is then released. This working model is consistent with the concept of the role of tumour microenvironment in the control of cells contained in a tumour. ${ }^{34}$ The treatment of ECs with SUT inhibits VE-cadherin tyrosine phosphorylation which then inhibits the cleavage and the release of sVE. This is not the case for TEM which is a serine-threonine kinase inhibitor. Thus, the use of sVE as a biomarker might be valuable in constructing individualised therapies for TKI treatments.

Of importance, we found that the level of sVE in $\mathrm{MRCC}$ patients at diagnosis is significantly higher than in HD group. This suggests an endothelial junction instability, since sVE is the adhesive part of VE-cadherin that should stay bound to the membrane to ensure vascular integrity. ${ }^{14}$ This result raises the question of the role of the soluble VE-cadherin in cancer. In metastatic breast cancer patients, we previously showed that a decrease of sVE level after 6 weeks of treatment was correlated with good prognosis. ${ }^{21}$ This result suggests that high levels of sVE might be involved in some cell signalling pathways and in tumour activation. This idea is supported by a recent report demonstrating that the extracellular domain of VE-cadherin contains two RGD motifs (specific for human), which are involved in invasion, proliferation and migration of tumour cells through the activation of a2 $\beta 1$ integrin. ${ }^{35}$ Further studies are required to better understand whether sVE in patients possesses tumour promoting functions.

In mRCC, several serum, urinary and plasma biomarkers have recently been described, ${ }^{36}$ including elevated circulating MMPs, ${ }^{37,38}$ as well as neovascularity. ${ }^{39}$ However, our study demonstrates for the first time the presence of sVE in the serum from RCC patients, as well as a SUT-cycle-dependent decrease of sVE level for patients who responded to the therapy. Interestingly, we observed that not all patients exhibited a decrease in sVE level after one cycle of SUT treatment and the reason for this is not known yet. One hypothesis is that the available concentration of the SUT in the bloodstream is different from one patient to another. As shown by therapeutic drug monitoring, the pharmacokinetics of SUT and its active metabolite are subjected to large interindividual variability, especially regarding bioavailability. ${ }^{40-42}$ These variations in the drug concentrations might explain differences in SUT effects on sVE levels in MRCC patients. Then, it would be interesting to study in the future the correlation of SVE levels and the concentrations of drugs in blood to provide information to the clinician in order to potentially adapt the dose of treatment. Furthermore, it would be worth considering a measurement of sVE every 6 weeks instead of one measurement after one cycle of SUT to ensure a better follow-up of mRCC patients.

For the patients treated with a combined therapy, including BEV and TEM or BEV and IFN, we found that the variations of sVE level after one cycle of treatment were not predictive of the response to the treatment. This result does not fit with our in vitro hypothesis (Fig. 6) as we could have expected that any therapy inhibiting VEGF-signalling pathway would have impaired SVE release. However, this result is of major importance as it shows that other potential activated signalling pathways might be involved in tumour progression. Indeed, in a study conducted in a genetic mouse model of neuroendocrine cancer, it was observed that blocking the VEGF pathway by anti-VEGF monoclonal antibody resulted in upregulation of several pro-angiogenicfactors, such as FGF-2, angiopoietin, VEGF, PDGF $\beta$, transforming growth factor a (TGF-a), erythropoietin (EPO), matrix metalloproteinase 1 (MMP-1), epidermal growth factor receptor (EGFR), hepatocyte growth factor receptor (HGFR/cMET), cyclin D1, stromal cell-derived factor 1 (SDF1) and its receptor CXC chemokine receptor 4 (CXCR4). ${ }^{43}$ As $B E V$ is a monospecific drug that blocks only VEGF-A, it does not affect all these actorsactors involved in the remodeling, perfusion, and metabolism of vessels in the tumour, which might participate to VE-cadherin modifications and to tumour escape from antiVEGF therapy.

SUT and TEM are among the first small molecule inhibitors used in kidney cancer $^{5}$ with some success, but also with some toxicity. ${ }^{37,44,45}$ In the TORAVA study, no benefit was noted in the BEV and TEM combination group ${ }^{44}$ and indeed that study showed that this combination therapy was toxic. Whether or not variations 
of SVE in patients treated by these combinations are related to toxicities remained to be explored. In contrast to BEV, SUT has the big advantage to inhibit several tyrosine-kinase activities that are overexpressed in cancer, which might explain its better efficacy than other combined therapies. ${ }^{46}$ Further evaluation of sVEcadherin levels in mRCC patients treated with other TKI is now needed in a prospective study. The quantification of sVE variations for each patient undergoing TKI treatment might provide personalised clinical information about toxicity or responsiveness to therapy, which would fit with the personalised medicine of the future.

\section{ACKNOWLEDGEMENTS}

We are indebted to Laurie Godart, Marie Courcon for technical assistance. We thank Pr Donald MARTIN (UGA-TIMC-IMAG-SyNABi team) for revising the English writing in the last version of this manuscript. We thank the investigators and the patients and their families who made this study possible. This work was supported by the French National Institute of Health and Medical Research INSERM (UMRS 1036), the French Atomic Energy and Alternative Energies Commission (CEA), Institut de Biosciences et Biotechnologies de Grenoble (BIG), Grenoble University Hospital, Institut National du Cancer (INCA 07/3D1616/PL-96-031/NG-NC) (I.V.), French Association against cancer (ARC Foundation), Federation Nationale des Centres de Lutte contre le Cancer (GEFLUC Grenoble-Dauphiné-Savoie) (IV), INCA Translationnel (2016-026, GP) Fondation ARC (GP) Fondation de France (GP), Fondation Mora (GP). Helena Polena and Abir Khalil-Mgharbel received grants from ARC Foundation (IV). Adama Sidibé received a grant from Courtin Arthritis Foundation (IV). The protocols TORAVA and SUVEGIL were approved by the ethics review boards of each participating institution and all patients provided informed consent for enrolment in the trial. These studies were sponsored by Centre Léon Berard and Centre Antoine Lacassagne, respectively, and registered in ClinicalTrials.gov (NCT00619268 for TORAVA, and NCT00943839 for SUVEGIL)

\section{AUTHOR CONTRIBUTIONS}

The study conception and design: I.V. conceived the research, and conducted the project. Acquisition of data: H.P., J.C., M.D., A.K.-M., A.D., C.R., and O.F. Analysis and interpretation: I.V., G.P., D.B., J.M.F., B.E., S.N. Analysis and interpretation of the biostatistics: M.D., G.P., J.-L.Q., E.B., C.S.-F., D.B., J.M.F., B.E., S.N., and I.V. Writingoriginal draft: H.P., J.C., M.D., A.S., G.P., and I.V. All authors were involved in revising the manuscript. Writing - review and editing: M.D., G.P., and I.V. All authors approved the final version to be published. Dr. Vilgrain had full access to all of the data in the study and takes responsibility for the integrity of the data and the accuracy of the data analysis. H.P., J.C., and M.D. contributed equally to the first authorship of this manuscript. I.V., and G.P. led the project.

\section{ADDITIONAL INFORMATION}

Supplementary information is available for this paper at https://doi.org/10.1038/ s41416-018-0054-5.

Competing interests: The authors declare no competing interest.

Note: This work is published under the standard license to publish agreement. After 12 months the work will become freely available and the license terms will switch to a Creative Commons Attribution 4.0 International licence (CC BY 4.0).

\section{REFERENCES}

1. Ferlay, J. et al. Cancer incidence and mortality worldwide: sources, methods and major patterns in GLOBOCAN 2012. Int. J. Cancer 136, E359-E386 (2015).

2. Afriansyah, A., Hamid, A. R., Mochtar, C. A. \& Umbas, R. Targeted therapy for metastatic renal cell carcinoma. Acta Med. Indones. 48, 335-347 (2016).

3. Yang, J. C. et al. A randomized trial of bevacizumab, an anti-vascular endothelial growth factor antibody, for metastatic renal cancer. N. Engl. J. Med 349, 427-434 (2003).

4. Escudier, B. et al. Axitinib versus sorafenib in advanced renal cell carcinoma: subanalyses by prior therapy from a randomised phase III trial. Br. J. Cancer 110, 2821-2828 (2014).

5. Motzer, R. J. et al. Sunitinib versus interferon alfa in metastatic renal-cell carcinoma. N. Engl. J. Med 356, 115-124 (2007).
6. Motzer, R. J. et al. Overall survival and updated results for sunitinib compared with interferon alfa in patients with metastatic renal cell carcinoma. J. Clin. Oncol. 27, 3584-3590 (2009).

7. Motzer, R. J., Escudier, B., Gannon, A. \& Figlin, R. A. Sunitinib: ten years of successful clinical use and study in advanced renal cell carcinoma. Oncologist 22, 41-52 (2017).

8. Huang, D. et al. Sunitinib acts primarily on tumour endothelium rather than tumour cells to inhibit the growth of renal cell carcinoma. Cancer Res. 70, 1053-1062 (2010).

9. Dufies, M. et al. Sunitinib stimulates expression of VEGFC by tumour cells and promotes lymphangiogenesis in clear cell renal cell carcinomas. Cancer Res. 77, 1212-1226 (2017a).

10. Giuliano, S. et al. Resistance to sunitinib in renal clear cell carcinoma results from sequestration in lysosomes and inhibition of the autophagic flux. Autophagy 11, 1891-1904 (2015).

11. Joosten, S. C. et al. Resistance to sunitinib in renal cell carcinoma: from molecular mechanisms to predictive markers and future perspectives. Biochim. Biophys. Acta 1855, 1-16 (2015).

12. Karashima, T. et al. Expression of angiogenesis-related gene profiles and development of resistance to tyrosine-kinase inhibitor in advanced renal cell carcinoma: characterization of sorafenib-resistant cells derived from a cutaneous metastasis. Int. J. Urol. 20, 923-930 (2013).

13. Dejana, E., Tournier-Lasserve, E. \& Weinstein, B. M. The control of vascular integrity by endothelial cell junctions: molecular basis and pathological implications. Dev. Cell 16, 209-221 (2009).

14. Bouillet, L. et al. Endothelial junctions: exploiting their instability in the development of biomarkers for vascular remodelling. Med. Sci. 30, 633-635 (2014).

15. Wallez, Y. et al. Src kinase phosphorylates vascular endothelial-cadherin in response to vascular endothelial growth factor: identification of tyrosine 685 as the unique target site. Oncogene 26, 1067-1077 (2007).

16. Lambeng, $\mathrm{N}$. et al. Vascular endothelial-cadherin tyrosine phosphorylation in angiogenic and quiescent adult tissues. Circ. Res. 96, 384-391 (2005).

17. Sidibé, A. et al. Dynamic phosphorylation of VE-cadherin Y 685 throughout mouse estrous cycle in ovary and uterus. Am. J. Physiol. Heart Circ. Physiol. 307, H448-H454 (2014).

18. Sidibé, A. et al. Soluble vascular-endothelial (VE)-cadherin: toward a marker of endothelial dysfunction. In: J. J. Feige, G. Pagès, F. Soncin (eds). Molecular Mechanisms of Angiogenesis (pp. 461-478. Springer-Verlag, France, 2014).

19. Sidibé, A. et al. Soluble VE-cadherin in rheumatoid arthritis patients correlates with disease activity: evidence for tumour necrosis factor a-induced VE-cadherin cleavage. Arthritis Rheum. 64, 77-87 (2012).

20. Vilgrain, I. et al. Evidence for post-translational processing of vascular endothelial (VE)-cadherin in brain tumours: towards a candidate biomarker. PLOS ONE 8 e80056, https://doi.org/10.1371/journal.pone.0080056 (2013).

21. Rochefort, P. et al. Soluble VE-cadherin in metastatic breast cancer: an independent prognostic factor for both progression-free survival and overall survival. Br. J. Cancer 116, 356-361 (2017).

22. Lê, J. et al. Quantitative zymography of matrix metalloproteinases by measuring hydroxyproline: application to gelatinases A and B. Electrophoresis 20, 2824-2829 (1999).

23. Dufies, M. et al. CXCL7 is a predictive marker of sunitinib efficacy in clear cell renal cell carcinomas. Br. J. Cancer 117, 947-953 (2017b).

24. Kluger, H. M. et al. Classification of renal cell carcinoma based on expression of VEGF and VEGF receptors in both tumour cells and endothelial cells. Lab. Invest. 88, 962-972 (2008).

25. Cunningham, D. et al. Randomised trial of irinotecan plus supportive care versus supportive care alone after fluorouracil failure for patients with metastatic colorectal cancer. Lancet 352, 1413-1418 (1998).

26. Figlin, R. A. Mechanisms of disease: survival benefit of temsirolimus validates a role for mTOR in the management of advanced RCC. Nat. Clin. Pract. Oncol. 5 601-609 (2008).

27. Parker, B. S., Rautela, J. \& Hertzog, P. J. Antitumour actions of interferons: implications for cancer therapy. Nat. Rev. Cancer 16, 131-144 (2016).

28. Gialeli, C., Theocharis, A. D. \& Karamanos, N. K. Roles of matrix metalloproteinases in cancer progression and their pharmacological targeting. FEBS J. 278, 16-27 (2011).

29. Senger, D. R. et al. Vascular permeability factor (VPF, VEGF) in tumour biology. Cancer Metastas. Rev. 12, 303-324 (1993).

30. Di Desidero, T. et al. Antiproliferative and proapoptotic activity of sunitinib on endothelial and anaplastic thyroid cancer cells via inhibition of Akt and ERK1/2 phosphorylation and by down-regulation of cyclin-D1. J. Clin. Endocrinol. Metab. 98, E1465-E1473 (2013).

31. Motzer, R. J. et al. Investigation of novel circulating proteins, germ line singlenucleotide polymorphisms, and molecular tumour markers as potential efficacy 
biomarkers of first-line sunitinib therapy for advanced renal cell carcinoma. Cancer Chemother. Pharmacol. 74, 739-750 (2014).

32. Kamiyama, H., Takano, S., Tsuboi, K. \& Matsumura, A. Anti-angiogenic effects of SN38 (active metabolite of irinotecan): inhibition of hypoxia-inducible factor 1 alpha (HIF-1alpha)/vascular endothelial growth factor (VEGF) expression of glioma and growth of endothelial cells. J. Cancer Res. Clin. Oncol. 131, 205-213 (2005).

33. Tien, W. S., Chen, J. H. \& Wu, K. P. Sheddome DB: the ectodomain shedding database for membrane-bound shed markers. BMC Bioinform. 18, 1465-1467 (2017).

34. De Palma, M., Biziato, D. \& Petrova, T. V. Microenvironmental regulation of tumour angiogenesis. Nat. Rev. Cancer 17, 457-474 (2017).

35. Bartolomé, R. A. et al. VE-cadherin RGD motifs promote metastasis and constitute a potential therapeutic target in melanoma and breast cancers. Oncotarget $\mathbf{8}$, 215-227 (2017).

36. Stubbs, C. et al. A study of angiogenesis markers in patients with renal cell carcinoma undergoing therapy with sunitinib. Anticancer Res. 37, 253-259 (2017).

37. Cohen, R. B. \& Oudard, S. Antiangiogenic therapy for advanced renal cell carcinoma: management of treatment-related toxicities. Invest. New Drug 30, 2066-2079 (2012).

38. Pastore, A. L. et al. Serum and urine biomarkers for human renal cell carcinoma. Dis. Markers 2015, 251403 (2015).
39. Bauman, T. M., Huang, W., Lee, M. H. \& Abel, E. J. Neovascularity as a prognostic marker in renal cell carcinoma. Hum. Pathol. 57, 98-105 (2016).

40. Blanchet, B. et al. Development and validation of an HPLC-UV-visible method for sunitinib quantification in human plasma. Clin. Chim. Acta 404, 134-139 (2009).

41. Sabanathan, D. et al. Dose individualization of sunitinib in metastatic renal cell cancer: toxicity-adjusted dose or therapeutic drug monitoring. Cancer Chemother. Pharmacol. 80, 385-393 (2017).

42. Cabel, L. et al. Drug monitoring of sunitinib in patients with advanced solid tumours: a monocentric observational French study. Fundam. Clin. Pharmacol. 32, 98-107 (2018).

43. Fernando, N. T. et al. Tumor escape from endogenous, extracellular matrixassociated angiogenesis inhibitors by up-regulation of multiple proangiogenic factors. Clin. Cancer Res 14, 1529-1539 (2008).

44. Négrier, S. et al. Temsirolimus and bevacizumab, or sunitinib, or interferon alfa and bevacizumab for patients with advanced renal cell carcinoma (TORAVA): a randomised phase 2 trial. Lancet Oncol. 12, 673-680 (2011).

45. Bhojani, N. et al. Toxicities associated with the administration of sorafenib, sunitinib, and temsirolimus and their management in patients with metastatic renal cell carcinoma. Eur. Urol. 53, 917-930 (2008).

46. Noé, G. et al. Clinical and kinomic analysis identifies peripheral blood mononuclear cells as a potential pharmacodynamic biomarker in metastatic renal cell carcinoma patients treated with sunitinib. Oncotarget 7, 67507-67520 (2016). 